\title{
Síndrome hipotalámico como presentación inusual de recaída en el sistema nervioso central de leucemia linfoblástica aguda en niños: reporte de 4 casos
}

\author{
Hypothalamic syndrome as an unusual presentation of CNS relapse of acute \\ lymphoblastic leukemia in children: report of 4 cases
}

\author{
Dra. Marina Mathey ${ }^{a}$, Dra. Myriam Guitter ${ }^{a}$, Dr. Jorge Rossi ${ }^{b}$, Dr. Antonio Latella ${ }^{c}$ y Dra. M. Sara Felice ${ }^{a}$
}

\begin{abstract}
RESUMEN
La leucemia linfoblástica aguda (LLA) es la patología oncológica más frecuente en pediatría, y corresponde al $23 \%$ de las neoplasias en menores de 15 años. Alrededor del $20 \%$ de los pacientes con LLA presentan recaídas, en la mayoría de los casos, en la médula ósea. Las recaídas extramedulares son inusuales y las dos localizaciones más frecuentes son el sistema nervioso central (SNC) y los testículos. Cuando las recaídas ocurren en el SNC, suelen manifestarse con un síndrome meníngeo. El síndrome hipotalámico se define como la presencia de hiperfagia, obesidad y / o cambios en el estado de ánimo, y es una forma de presentación clínica inusual de las recaídas en el SNC y debe alertar al pediatra para mantener un alto índice de sospecha.

Se describen cuatro casos que se presentaron con síndrome hipotalámico al momento de desarrollar una recaída de LLA en el SNC.

Palabras clave: enfermedades hipotalámicas, leucemia linfoblástica aguda, pediatría.
\end{abstract}

\begin{abstract}
Acute lymphoblastic leukemia (ALL) is the most common malignancy in childhood, corresponding to $23 \%$ of cancer in children younger than 15 years old. About $20 \%$ of ALL cases will relapse, commonly in the bone marrow. Extramedullar relapses are unusual, and the two most frequent locations are CNS and testicles. ALL relapses, when diagnosed in the CNS, frequently present with clinical features of a meningeal syndrome. The hypothalamic syndrome, consisting of hyperphagia, obesity and / or behavior disturbances, corresponds to an unusual presentation of relapses in this location and should alert pediatricians to suspect it.

We describe 4 ALL cases of hypothalamic syndrome at the time of CNS relapse.

Key words: hypothalamic diseases, acute lymphoblastic leukemia, pediatrics.
\end{abstract}

a. Servicio de Hematología y Oncología.

b. Servicio de Inmunología y Reumatología.

c. Centro de atención integral del pacientehemato-oncológico. Hospital de Pediatría S.A.M.I.C. "Prof. Dr. Juan P. Garrahan”, Ciudad Autónoma de Buenos Aires, Argentina.

Correspondencia:

Dra. Marina Mathey: mmathey.mm@gmail.com

Financiamiento: Ninguno.

Conflicto de intereses: Ninguno que declarar.

Recibido: 20-7-2020

Aceptado: 29-12-2020 http: / / dx.doi.org/10.5546/ aap.2021.e242

Cómo citar: Mathey M, Guitter M, Rossi J, Latella A, Felice MS. Síndrome hipotalámico como presentación inusual de recaída en el sistema nervioso central de leucemia linfoblástica aguda en niños: reporte de 4 casos. Arch Argent Pediatr 2021;119(3):e242-e246.

\section{INTRODUCCIÓN}

La leucemia linfoblástica aguda (LLA) es la patología oncológica más frecuente en pediatría y corresponde aproximadamente al $23 \%$ de las enfermedades malignas en menores de 15 años. ${ }^{1,2}$ En nuestro país, según el Registro Oncopediátrico Hospitalario Argentino (ROHA), cada año se presentan de 30 a 34 casos nuevos de LLA por millón de niños menores de 15 años, y cerca de 400 nuevos casos por año. ${ }^{3}$

La LLA pediátrica tiene buen pronóstico, con una sobrevida global del $80 \%$, aproximadamente. Hoy en día, en Argentina, la sobrevida libre de eventos es de 70-80\%. La recaída de la enfermedad es la principal causa de fracaso del tratamiento, se presenta en aproximadamente el $20 \%$ de los pacientes y, en la mayoría de los casos, se localiza en la médula ósea (MO) (recaída hematológica). Las recaídas extramedulares son inusuales y las dos localizaciones más frecuentes son el sistema nervioso central (SNC) y los testículos. ${ }^{1}$

En el momento del diagnóstico, el compromiso de SNC se presenta en menos del $5 \%$ de las LLA de subtipo precursor B, y entre el 10-15\% de las LLA subtipo precursor T. Los factores de riesgo predisponentes para el compromiso del SNC (tanto inicial como en la recaída) son: inmunofenotipo $\mathrm{T}$, recuento inicial de leucocitos elevado (hiperleucocitosis), edad menor de 1 año al diagnóstico y la presencia del gen de fusión TCF3-PBX1 de la t $(1 ; 19) .{ }^{1,2}$

Tanto el compromiso inicial como la recaída de la LLA en el SNC pueden resultar un hallazgo en pacientes asintomáticos en quienes se realiza 
una punción lumbar (PL) con análisis del líquido cefalorraquídeo (LCR). Cuando la presentación es sintomática, los cuadros clínicos más frecuentes son el síndrome meníngeo o la hipertensión endocraneana con cefalea, convulsiones, vómitos, compromiso focal neurológico y/o alteración en el fondo de ojo. ${ }^{1}$

El compromiso del SNC se define por la presencia de $>5$ células $/ \mu \mathrm{L}$ en el LCR con confirmación de la presencia de blastos en una muestra de PL no traumática, presencia de lesiones en el cerebro o las meninges en estudios por imágenes (tomografía computada y resonancia magnética nuclear), alteración en los pares craneanos no relacionada con otro origen, y / o compromiso retiniano en el fondo de ojo. ${ }^{1,2}$

El síndrome hipotalámico, también conocido como obesidad hipotalámica, se define por la presencia de hiperfagia, obesidad y / o cambios en el estado de ánimo y corresponde a una forma de presentación clínica inusual de la LLA. Su fisiopatogenia podría estar relacionada con la infiltración o destrucción de los núcleos ventromediales (centro de la saciedad) y / o paraventriculares (eje corticotropo) del hipotálamo, lo que causa hiperfagia (apetito insaciable), incremento de peso patológico y/o diabetes insípida. ${ }^{1,4}$ En este artículo se presenta la experiencia de los autores con cuatro pacientes con diagnóstico LLA en quienes el síndrome hipotalámico fue la primera manifestación de la recaída en el SNC, y se revisa la bibliografía sobre este tipo de hallazgos inusuales.

\section{PRESENTACIÓN DE CASOS CLÍNICOS}

Se presentan cuatro casos de niños con un rango de edades de entre 2 y 9 años (dos niños y dos niñas) con diagnóstico previo de LLA de subtipo precursor B sin alteraciones citogenéticas recurrentes, sin compromiso inicial de SNC (SNC1) y sin factores de riesgo. Solo uno de los pacientes había presentado un evento adverso (una recaída temprana aislada en $\mathrm{MO}$ ) a los 29 meses de la primera remisión completa (RC). Ninguno de los pacientes presentaba compromiso de SNC ni pertenecía al grupo de alto riesgo en la estadificación inicial, por lo que recibieron quimioterapia intratecal profiláctica con metotrexato y no tenían indicación de recibir radioterapia en su primer tratamiento de acuerdo con los protocolos administrados. El paciente con antecedente de la recaída en la $\mathrm{MO}$ tampoco recibió radioterapia en el SNC en su segundo tratamiento.
Tres de los pacientes se encontraban en la primera $\mathrm{RC}$ (a 18, 20 y 33 meses) al momento del inicio de los síntomas, y uno de ellos, en la segunda RC (a 17 meses).

Tres pacientes presentaron recaída aislada en el SNC, mientras que uno de ellos presentó una recaída combinada en la MO y el SNC.

Los cuatro pacientes presentaron hiperfagia e incremento patológico del peso (entre $7 \mathrm{~kg} \mathrm{y}$ $20 \mathrm{~kg}$ en un lapso de 2 a 6 meses). Uno de ellos presentó cambios en el humor (irritabilidad) y otro, cambios en la conducta. Los síntomas neurológicos acompañantes fueron disminución de la agudeza visual en dos los pacientes y dolor lumbar asociado a dificultad para la micción en el paciente que presentó, además, compromiso de la médula espinal. En el paciente que presentó recaída combinada se observó pancitopenia y hepatoseplenomegalia.

Solo un paciente presentó, en los estudios por imágenes, hallazgos compatibles con compromiso del SNC, con infiltración en el fondo del globo ocular izquierdo (desprendimiento de retina) e infiltración leptomeníngea que se extendía al filum terminale y a la cola de caballo, acompañados de manifestaciones clínicas acordes (Figura 1). Los otros pacientes presentaban imágenes inespecíficas.

En tres pacientes se efectuó el diagnóstico con la realización de PL; el LCR mostró pleocitosis (430, 432 y 1400 células $\left./ \mathrm{mm}^{3}\right)$. En el conteo realizado en cámara, se observaron elementos que correspondían morfológicamente a linfoblastos, y se confirmó que se trataba de precursores $\mathrm{B}(\mathrm{CD} 19+, \mathrm{CD} 22+, \mathrm{CD} 10+, \mathrm{CD} 56+$, $\mathrm{CD} 45+$ y CD20+) por citometría de flujo. En un paciente fue necesario realizar una biopsia de leptomenínges debido a que presentaba bloqueo del canal medular, lo que impidió la obtención de LCR mediante PL; los resultados de anatomía patológica confirmaron el diagnóstico.

Todos los pacientes recibieron tratamiento para recaída de LLA: dos alcanzaron una segunda $\mathrm{RC}$, uno no respondió al tratamiento y falleció por progresión de la enfermedad, y el cuarto paciente falleció durante la fase de inducción por sangrado en SNC como complicación. De los dos pacientes que alcanzaron una segunda RC, uno de ellos desarrolló una segunda enfermedad maligna, un tumor neuroectodérmico primitivo supratentorial (PNET, por sus siglas en inglés) según la clasificación de la Organización Mundial de la Salud (OMS) de 2007 (no se contó con el taco para reclasificarlo según los parámetros de la 
OMS de 2016)y falleció posteriormente debido a la progresión del mismo; el otro paciente presentó

FIGURA 1. Hallazgos compatibles con compromiso neurológico en la resonancia magnética nuclear. (A) ponderada en T1: ocupación del espacio aracnoideo espinal perimedular y adyacente al cono, con refuerzo poscontraste (infiltración leptomeningea) y extensión al filum terminale y a la cola de caballo; (B) tejido con señal isointensa en T1, refuerzo con contraste por vía intravenosa en el fondo del globo ocular izquierdo (desprendimiento de retina)

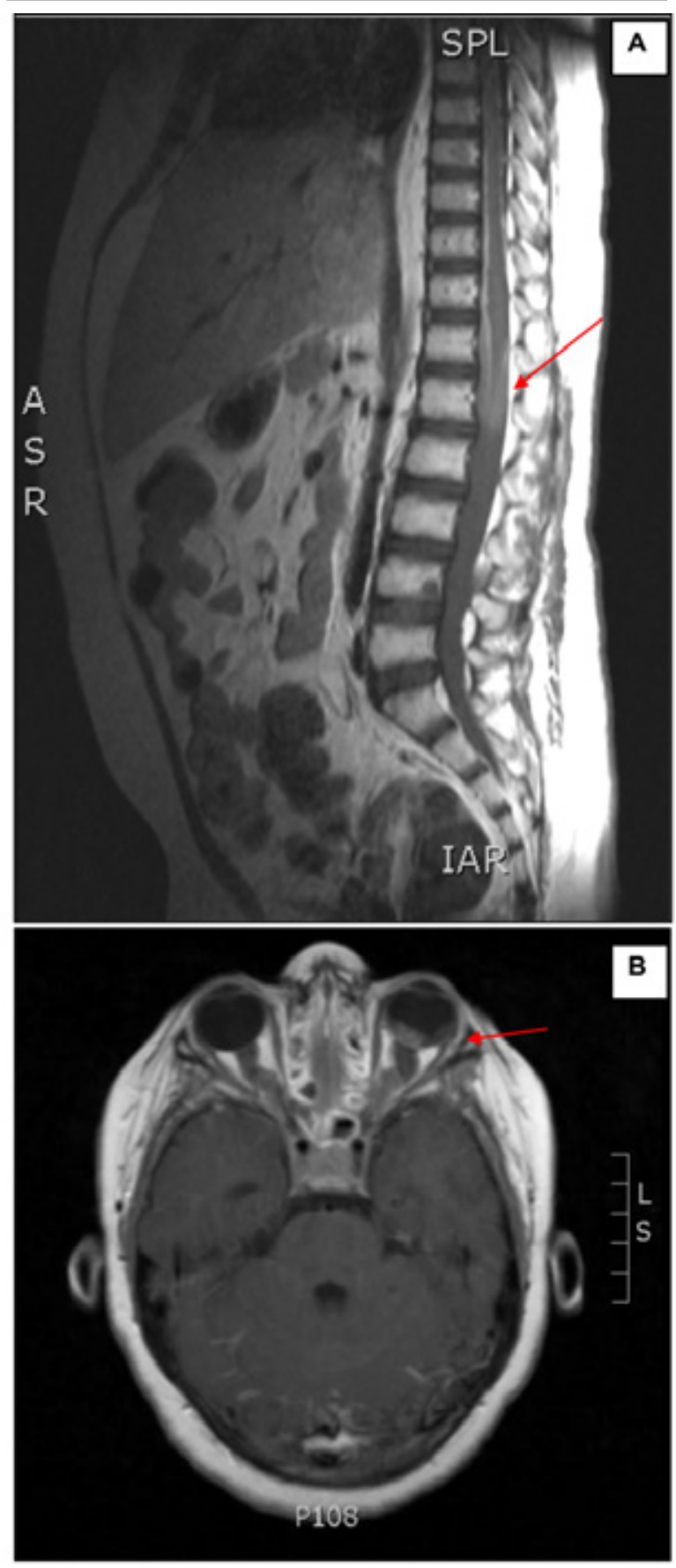

una segunda recaída de LLA en el SNC a los ocho meses de la segunda $\mathrm{RC}$ y falleció por progresión de enfermedad.

\section{DISCUSIÓN}

A partir del análisis de estos cuatro casos que presentaron una recaída de LLA en el SNC con una forma de presentación inusual, se plantean dos mecanismos fisiopatológicos que justificarían el síndrome clínico, también llamado de obesidad hipotalámica: ${ }^{1,4}$

1. La disfunción hipotalámica anterior (paraventricular) por infiltración leucémica puede provocar un déficit en el eje corticotropo, con alteración en el sistema de la hormona liberadora de corticotropina (CRH); esto causa un aumento de la secreción de hormona adrenocorticotrópica (ACTH) por la hipófisis y un exceso de cortisol periférico de origen hipofisario (con manifestación clínica de síndrome de Cushing). ${ }^{4}$

2. El daño (por destrucción o infiltración) de los núcleos mediales del hipotálamo, donde se encuentra el centro de la saciedad. Los más afectados suelen ser los núcleos arcuato, paraventricular, ventromedial, dorsomedial y dorsal. El compromiso resulta en una incapacidad para integrar diversas señales aferentes de hormonas y neuropéptidos. El mecanismo fisiopatogénico no se conoce bien, probablemente es multifactorial y causa desregulación de la saciedad, aumento de la ingesta (hiperfagia) y, en consecuencia, obesidad. $^{5}$

Entre las causas más frecuentes de síndrome hipotalámico se encuentran las lesiones directas en el SNC (debidas a irradiación, cirugías o traumatismos) o las enfermedades propias del eje hipotálamo-hipofisario, como algunas enfermedades congénitas (síndrome de Prader-Willi o síndrome de Laurence-MoonBiedl) o tumorales (adenomas hipofisarios, craneofaringiomas, tumores germinales de SNC, hamartomas). La leucemia se encuentra dentro de las causas infrecuentes de este síndrome. ${ }^{4-6}$

Existen escasas publicaciones acerca del síndrome hipotalámico como manifestación de compromiso del SNC en la LLA. En una búsqueda bibliográfica, se encontraron solo ocho informes de casos pediátricos en inglés (Tabla 1): siete sobre $\operatorname{LLA}^{7-13}$ y uno sobre linfoma linfoblástico de tipo $\mathrm{B}_{r^{14}}$. Todos ellos, en coincidencia con los cuatro casos aquí descritos, presentaron clínica de hiperfagia $(6 / 8)$, incremento patológico de peso 
(7/8) y / o alteraciones del humor (5/8). ${ }^{7-14}$

Siete de ellos habían alcanzado la RC al momento del inicio del cuadro clínico, con el síndrome hipotalámico como manifestación clínica de recaída de la enfermedad ${ }^{7-13} \mathrm{y}$, en un paciente, fue la manifestación inicial al diagnóstico. ${ }^{14}$

En seis de los pacientes se realizó PL. En cinco se hallaron elementos que correspondían a linfoblastos de estirpe B y, en un paciente, aumento de la presión de apertura del LCR.7-14

Si bien el síndrome hipotalámico es una forma de presentación inusual, se debe sospechar su presencia ante cuadros de hiperfagia, aumento patológico de peso y/o cambios en el estado de ánimo como una posible manifestación del compromiso de SNC en pacientes con LLA, como fue el caso de los niños descritos.

TABla 1. Casos de síndromes hipotalámicos en leucemia linfoblástica aguda y linfoma descritos en la literatura

\begin{tabular}{|c|c|c|c|c|c|c|c|}
\hline $\begin{array}{l}\text { Autor } \\
\text { (año) }\end{array}$ & $\begin{array}{l}\text { Edad } \\
\text { (años) }\end{array}$ & Sexo & $\begin{array}{c}\text { Tipo de } \\
\text { Leucemia }\end{array}$ & $\begin{array}{c}\text { Estatus } \\
\text { oncológico }\end{array}$ & $\begin{array}{c}\text { Tratamiento } \\
\text { previo en SNC }\end{array}$ & $\begin{array}{l}\text { Presentación } \\
\text { clínica }\end{array}$ & LCR \\
\hline $\begin{array}{l}\text { Bastrup-Madsen } \\
(1963)^{7}\end{array}$ & 2 & M & LLA & $\begin{array}{l}\text { Segunda RC } \\
\text { (4 meses) }\end{array}$ & No & $\begin{array}{l}\text { Hiperfagia } \\
\text { Incremento de peso } \\
\text { (9,3 kg en } 4 \text { meses) } \\
\text { Cambio de ánimo: } \\
\text { impaciente por la comid }\end{array}$ & $\begin{array}{l}\text { PL no realizada } \\
\text { da }\end{array}$ \\
\hline Barak $(1968)^{8}$ & 4 & M & LLA & $\mathrm{RC}$ & No & $\begin{array}{l}\text { Hiperfagia } \\
\text { Incremento de peso ( } 4 \mathrm{k} \\
\text { Comportamiento bizarro } \\
\text { (ira, llanto inexplicado) } \\
\text { Leucocitosis, adenomeg }\end{array}$ & $\begin{array}{l}\text { PL no realizada } \\
\mathrm{kg}) \\
\text { ro } \\
\text { galias }\end{array}$ \\
\hline Al-Rashid (1971) ${ }^{9}$ & 4 & $\mathrm{~F}$ & LLA & $\mathrm{RC}$ (8 meses) & No & $\begin{array}{l}\text { Incremento de peso } \\
\text { (6,6 kg en } 2 \text { meses) } \\
\text { Papiledema } \\
\text { Escasos blastos }\end{array}$ & $\begin{array}{l}\text { Presión de } \\
\text { apertura } \\
\text { aumentada }\end{array}$ \\
\hline Tilley $(1989)^{10}$ & 5 & M & LLA & RC (24 meses) & $\begin{array}{c}\text { QT IT: } \\
\text { metotrexato } \\
\text { RDT: } 18,08 \text { Gy }\end{array}$ & $\begin{array}{l}\text { Incremento de peso } \\
\text { Cambios de ánimo }\end{array}$ & $\begin{array}{l}\text { Presencia } \\
\text { de blastos }\end{array}$ \\
\hline Ritchey $(2000)^{11}$ & 4 & $\mathrm{~F}$ & LLA & Segunda RC & $\begin{array}{c}\text { QT IT: } \\
\text { metotrexato } \\
\text { + citarabina } \\
\text { + hidrocortisona } \\
\text { realizó RDT en SNC }\end{array}$ & $\begin{array}{l}\text { Hiperfagia } \\
\text { Hipertensión } \\
\text { endocraneana }\end{array}$ & $\begin{array}{l}\text { Presencia } \\
\text { de blastos }\end{array}$ \\
\hline Satyanarayana $(2006)^{12}$ & ${ }^{12} 13$ & M & $\begin{array}{c}\text { LLA } \\
\text { (3 años de edad) } \\
\text { Recaída } \\
\text { testicular y } \\
\text { hematológica } \\
(9 \text { años })\end{array}$ & $\begin{array}{l}\text { Segunda RC } \\
\text { (30 meses) }\end{array}$ & $\begin{array}{l}\text { No realizó } \\
\text { RDT en SNC }\end{array}$ & $\begin{array}{l}\text { Hiperfagia } \\
\text { Incremento de peso } \\
\text { (20 kg en } 3 \text { meses) } \\
\text { Cambios abruptos } \\
\text { de comportamiento } \\
\text { Cefalea }\end{array}$ & $\begin{array}{c}131 \text { elementos } / \mathrm{mm}^{3} \\
(89 \% \text { de blastos) }\end{array}$ \\
\hline Li-Dan Zhang (2012) ${ }^{13}$ & 136 & M & $\begin{array}{l}\text { LLA preB } \\
\text { SNC1 }\end{array}$ & RC (24 meses) & $\begin{array}{c}\text { QT IT: } \\
\text { metotrexato } \\
+ \text { dexametasona }\end{array}$ & $\begin{array}{l}\text { Hiperfagia } \\
\text { Incremento de peso } \\
\text { (11 kg en } 3 \text { meses) } \\
\text { Cambios de humor: } \\
\text { introvertido, taciturno } \\
\text { CD19+, CD79a+ }\end{array}$ & $\begin{array}{c}18 \times 10^{6} \\
\text { elementos } / \mathrm{L} \\
\text { CF: CD22+, } \\
\text { HLA-DR+, } \\
\text { CD10+, CD34+, }\end{array}$ \\
\hline Quigg $(2012)^{14}$ & 3 & M & $\begin{array}{l}\text { LNH: linfoma } \\
\text { linfoblástico B }\end{array}$ & $\begin{array}{l}\text { Diagnóstico } \\
\text { inicial }\end{array}$ & -- & $\begin{array}{l}\text { Hiperfagia } \\
\text { Incremento de peso } \\
(14,5 \text { kg en } 6 \text { meses })\end{array}$ & $\begin{array}{c}788 \text { elementos } / \mathrm{mm}^{3} \\
\text { CF: CD10+, CD19+, TdT+ } \\
\text { Inmunohistoquímica: } \\
\text { PAX5+, TdT+ }\end{array}$ \\
\hline
\end{tabular}

M: masculino; F: femenino; RC: remisión completa; SNC: sistema nervioso central; SNC1: sin compromiso de SNC;

LLA: leucemia linfoblástica aguda; LLA preB: leucemia linfoblástica aguda subtipo precursor B; LNH: linfoma no Hodgkin; QT IT: quimioterapia intratecal; RDT: radioterapia; Gy: grays; CF: citometría de flujo. 
La obtención de LCR por PL es una técnica sencilla, que permite el análisis mediante citometría de flujo de los blastos leucémicos para confirmar el diagnóstico en forma precisa.

Desafortunadamente, el pronóstico de las recaídas de la LLA en SNC es malo, y está marcado por la dificultad de erradicar la enfermedad de esta localización, aún con el agregado de radioterapia al tratamiento (que, además, aumenta el riesgo de desarrollo de segundas neoplasias, como fue el caso de uno de los pacientes aquí descritos).

\section{REFERENCIAS}

1. Rabin K, Gramatges M, Margolin J, Poplack D. Acute Lymphoblastic leukemia. In: Pizzo PA, Poplack DG. Principles and practice of Pediatric Oncology. 7th ed. Philadelphia: Wolters Kluwer; 2016.Págs.480-491.

2. Swerdlow SH, Campo E, Harris NL, Jaffe ES, et al. WHO Classification of Tumours of Haematopoietic and Lymphoid Tissues. $4^{\text {th }}$ ed. Lyon, France: IARC Press; 2008.

3. Moreno F, Chapli MA. Registro Oncopediátrico Hospitalario Argentino. Tendencia de Incidencia 20002016 Supervivencia a 5 años 2005-2011 Tendencia de Supervivencia secular: 2000-04, 2005-09 y 2010-14. 6ta ed. Ciudad Autónoma de Buenos Aires: Instituto Nacional del Cáncer, 2018.

4. Sucunza Alfonso N, Barahona MJ, Webb SM. Síndromes hipotalámicos. Etiopatogenia. Manifestaciones clínicas. Estrategias diagnósticas. Medicine (Barc., Internet). 2008; 10(13):823-8.
5. Abuzzahab MJ, Roth CL, Shoemaker AH. Hypothalamic Obesity: Prologue and Promise. Horm Res Paediatr. 2019; 91(2):128-36.

6. Kim JH, Choi J. Pathophysiology and clinical characteristics of hypothalamic obesity in children and adolescents. Ann Pediatr Endocrinol Metab. 2013; 18(4):161-7.

7. Bastrup-Madsen P, Greisen O. Hypothalamic obesity in acute leukaemia. Report of a case. Acta Haematol. 1963; 29:109-16.

8. Barak Y, Liban E. Hypothalamic hyperphagia, obesity and disturbed behaviour in acute leukemia. Acta Paediatr Scand. 1968; 57(2):153-6.

9. Al-Rashid RA.Hypothalamic syndrome in acute childhood leukemia. Clin Pediatr (Phila). 1971; 10(1):53-4.

10. Tilley E, Ryalls MR, Williams MP. Ct demonstration of pituitary stalk relapse in childhood acute lymphoblastic leukaemia. Clin Radiol. 1989; 40(6):634-5.

11. Ritchey AK, Pollock BH, Lauer SJ, Andejeski Y, et al. Improved survival of children with isolated CNS relapse of acute lymphoblastic leukemia: a pediatric oncology group study. J Clin Oncol. 1999; 17(12):3745-52. [published correction appears in J Clin Oncol. 2000; 18(3):703].

12. Satyanarayana S, Cummings EA, Fernandez CV, Barnard D. Hyperphagia following childhood acute lymphoblastic leukemia: a symptom worth heeding. Clin Pediatr. 2006; 45(3):271-3.

13. Zhang LD, Li YH, Ke ZY, Huang LB, Luo XQ. Obesity as the initial manifestation of central nervous system relapse of acutelymphoblasticleukemia: Case report and literature review. J Cancer Res Ther. 2012; 8(1):151-3.

14. Quigg TC, Haddad NG, Buchsbaum JC, Shih CS. Hypothalamic obesity syndrome: rare presentation of $\mathrm{CNSp}$ B-Cell lymphoblasticlymphoma. Pediatr Blood Cancer. 2012; 59(5):930-3. 